\title{
ICTs and Music in Sensory and Motor Disabilities
}

\author{
https://doi.org/10.3991/ijes.v5i2.6386 \\ Paraskevi Theodorou \\ National Center for Scientific Research "Demokritos", Agia Paraskevi, Attica, Greece \\ van 4 . theodoroudgmail.com \\ Athanasios S. Drigas \\ National Center for Scientific Research "Demokritos", Agia Paraskevi, Attica, Greece \\ dr@iit.demokritos.gr
}

\begin{abstract}
With the advent of technology, the use of Information and Communication Technologies (ICTs) is essential for the improving of the quality of life of learners with Sensory and Motor disabilities. In this paper we present an overview of the most representative studies of the last decade (20052015), which deal with the two important issues in the field of ICT's, music and special education. The studies chosen will be classified according to the areas of needs they serve.
\end{abstract}

Keywords - Sensory and Motor disabilities, Visually, Deaf and Hearing Impaired people

\section{Introduction}

In the following papers there are presentations of the ways the visually impaired can satisfy their goals and needs on music [1]. One methodological approach for teaching music to blind children in Brazil is based on the interaction with the Musibraille software [2]. There is also a novel approach which explores on methods of converting printed eastern music notations to readable and controllable digitized output [3]. Another proposition is a music composition software named "Musical Composition Assistant" designed specifically for the people with physical and visual disabilities [4]. Next a user's study on the benefits and drawbacks of simultaneous spatial sounds in auditory interfaces for visually impaired and blind computer users is examined thoroughly [5]. A model to design multimedia software and virtual environment for blind learners is presented, using 3D auditory representations [6]. A detailed structure is presented with an integrated tele-informing and tele-education system (web portal) [7]. There is an analysis of the commonly used current technologies with the basic priority in accessibility and educating applications (ProTools, Cubase, Sibelius) [8]. A popular tabular format, which needs a certain algorithm to support it, still poses huge problems for screen readers because of diversity and complexity of the cells content [9]. The first humans to take advantage of autonomous mobile robots 
will be disabled ones and old-aged ones [Aylett, 2002]. Mobile robots will open radical revolutionary dimensions in the $21^{\text {st }}$ century [10].

As far as acoustical impaired individuals are concerned there is a design and evaluation of a system (Haptic Chair) they have developed to enhance the musical experience for the deaf [11]. A special emphasis is given in captioning and subtitling simultaneously with sign language [12]. A prototype system, supporting only the Portuguese sign language alphabet, was developed - the Kinect-Sign [13]. A computerassisted music-learning system (CAMLS) has been developed to help the hearing impaired practice playing a musical melody [14]. The next review concerns the technological, biological and acoustical constraints that limit music perception in Cochlear Implants (CI) users [15]. An investigation of music perception between deaf children and normal hearing ones is examined [16]. An intelligent web based system of four components: interaction, pedagogical, student and expert module aims to enhance concepts and skills of digital circuits for deaf students [17]. The last article of acoustical category describes a development work whose focus examines whether designing a game as an educational technology can contribute to education of deaf children in learning a language [18].

Concerning physical disabilities, one of the primary goals of the articles is to explore what difficulties instrumental music directors face when attempting to integrate students with physical disabilities into mainstream music education with the aid of technology [19]. The results of a case study are illustrated, where disabled students use the multimedia tool called PLAIME (Platform for the integration of handicapped children in music education) [20]. Bearing in mind the expanding popularity of virtual communities on massively multiplayer online games could be extensively effective particularly for the disabled people, eg the novel 'Snap Clutch' software [21]. The lack of physical skills of children with severe physical disabilities, so that they can play musical instruments or even play with their toys, make us know that it is of uttermost importance the use of a device to help them (MTM system) [22]. A lot of adapted interfaces are manufactured for typical users or disabled ones to play music form digital audio (AE2M project) [23]. An outstanding technological revolution is an ongoing process, particularly in robotics and Virtual Reality [24]. Playing a guitar or other musical instruments requires flexible and functional hands. Here there is an investigation of alternative interactions to enable or re-enable students with nonfunctional hands or arms to play a guitar [25].

\section{Sensory and Motor Disabilities}

\subsection{Visually Impaired Learners}

The next category concerns visually impaired individuals. The basic aims of the first research are studying the performance of a musical score and distinguish auditory symbols [1]. Audio format was approached as the most feasible and effective readable format to read music notations for visually impaired. The researchers have developed western music techniques. The Western music is more structural than the eastern, 
therefore is less confused than Eastern. "Adaptive Music Score Trainer" delivers a non visual presentation of music notations of Eastern music. This means that visually impaired will be easily attached to "it". The basic aims are studying the performance of a musical score and distinguish auditory symbols. As a result this study is very important, because it has revealed the problem of visualizing music notations. Concluding this adaptive music score trainer of visually impaired can be extended towards a music score generator which assist user to create a visual script of music notations from an audio input [1].

A good idea is to use Braille Music techniques as they are fully accessible to blind students working together with non blind children [2]. There are two approaches. First the blind musician uses sequence programs. In the second approach they use computer music editors. Among the programs that can be used, are Dancing Dots, a Braille Music Translator, which can convert files from MIDI and Music XML files than can be exported from standard software like Finale and Sibelius into Braille. Another interesting option is the Italian program Braille Music Editor (BME) that can edit scores directly or imported from Finale. Nowadays access is available with Dosvox and other tools like Jaws and NVDA. The main idea is that computers are applicable to different audiences. That means that during the initial period of learning, blind and sighted people can cooperate and learn together, as the accessibility of the software is satisfactory. The future of this research involves the evaluation of this technology and methodology to disseminate Braille music education in Portuguese language countries in Africa [2].

Some novel systems for music score recognition are called OMR, and it is similar to Optical Character Recognition (OCR) except that OMR is used to recognize musical symbols instead of letters [3]. Audio format has been identified as the most optimal readable format among other possible non-visual output formats such as hepatic/kinesthetic, gustatory and olfactory. Additionally the process is carried out in three main phases: 1. Zoning and Segmentation of the image of sheet music, 2. Adaptive OMR - Recognition of atomic symbols, 3. Converting into the readable format (audio format). Referring to the limitations of the research prototype has been only designed for visually impaired people, those who do not have multiple disabilities. Concluding students were very enthusiastic about using the system to study music notations as it helps to enhance the speed of learning [3].

Music composition software has a primary function allowing individuals unable to operate a traditional keyboard and mouse to compose music via a single-switch or a double-switch mode [4]. Specifically, it has a sounding component for the blinded or visually disadvantaged users. The former individuals with the hearing disadvantages must collaborate in order to navigate within the virtual environment. They do not need to see the screen to compose the music. Moreover users with severe physical disabilities can use an eye-tracking or blink-input device as an optional tool. The interface of the software is based on a digital MIDI music composition which also includes an autosave function and autoscan mode. Also, a scanning keyboard for Chinese input has been developed. Unlike the traditional digital phonetic table, this system provides more independence [4]. 
Two different auditory interfaces in spatial and non-spatial condition were proposed to represent the hierarchical menu structure of a simple word processing application [5]. The two main goals of the present research are: a) an effective auditory presentation of the hierarchical menu structures of a typical MS Windows application and b) a comparison among similar auditory interfaces with and without spatial sound in a controlled environment. The initial hypothesis that the spatial auditory interfaces with multiple simultaneous sounds should prove to be faster and more efficient than non spatial ones, was not confirmed. Multiple sounds can be perceived and understood if originating from different spatial positions. This phenomenon is called "Cocktail Party Effect" and is referred to the human ability to filter several simultaneous sounds. For everyday computer tasks, a hybrid auditory interface might be appropriate, in which the use of simultaneous spatial sounds can be turned on or off [5].

The particular method uses graphics, sounds and texts (multimedia) concerning sighted learners, whereas nontraditional interaction modes such as haptic devices are used for visually and sensory disabled people [6]. To represent geometric shapes, it is necessary to build a two-dimensional sound space. AudioDoom allows blind children to explore and interact with virtual worlds. Moreover, VirtualAurea is a set of spatial sound editors. The research project called "Blind Interactive Guide System" is based on radio frequency identification technology to provide information needed for navigating inside rooms. Another project aims to enrich the lives of people who are blind by developing visually perceptive wearable computers. Concluding scientists transfer the real world into appropriate computer representations with software like Theo and Seth, AudioChile and AudioVida, allowing users to make a precise mapping of the virtual environment and thus improving the interaction [6].

In web portal which is an integrated tele-informing and tele-education system, particular emphasis was given to the special access methods to create a user-friendly environment for users [7]. This portal, which is the first in Greece for disabled people, has to comply with the principles of "Universal Accessibility", "Assistive Technology" and "Design for All". In addition, this project aims at the development of a distance learning and tele-education system. The usage of multimedia applications is upgraded. The access methods include audio navigation, the possibility of enlarging the text and it is also addressed to normal citizens. Finally the visually impaired individual is navigated through audio messages (Real Audio) to audio information only [7].

Some programs like ProTools are particularly inaccessible environment for visually impaired (VI) users to work with. An alternative experience can be offered using computers running popular software (Cubase, Sibelius, etc.) with perhaps a small collection of 'plug-in' virtual instruments [8]. For visually impaired students, in particular, highly complex graphic environments simply do not let themselves to access via screen-reading software such as JAWS, HAL, MIDI Creator, E-Scape and MIDI Grid. Graphical notation of this type is difficult for visually impaired students to access and presents challenges for individuals with dyslexia. What is more there are two contemporary music performance technologies that also make use of gesture-based 
interaction: Soundbeam and the Benemin, methods of making music performance to disabilities like cerebral palsy issues and profound mobility problems [8].

The goal of this study with tabular data was to examine human performance and an accuracy of solving the most common tasks dealt with the virtual table composed of 100 cells [9]. There is still the question of access the numerical data in a quick way in the absence of visual feedback. A certain algorithm is presented to support an exploration of the tabular data in the absence of visual feedback. The majority of research has been based on the cross modal data transformation, on the use of sonification and musical techniques to convert musical information into auditory phonological representations. Extended research has been undertaken to convert visual presentation into another perceptual modality [9].

Blind children and visually impaired ones will be benefited by the emerging improvements of mobile robots in combination with computer interactions [10]. Specifically, Sony Aibo has produced a suitably modified robot named Miranda. Aibo stimulates blind toddlers and then leads them to interaction and gradually into engagement. Certainly there are a few questions and obstacles for blind toddlers using Miranda. Therefore certain modifications are vital for an effective and easy operation and use. Furthermore, toddlers strongly need to play, so meaningful play is learning with the valuable help of Miranda. Even the use of musical instruments will stimulate and motivate blind children or toddlers to interact and then learn. Above all there are open horizons at the intersection of human-computer interaction and robotics [10].

\subsection{Deaf and Hearing Impaired Learners}

A system which combines tactile and visual information might be used to enhance musical experience for the hearing impaired and improve the sense of hearing by enhancing sensory input of information via channels other than in-air audio reception by the ear, appropriate for acoustical disabled people [11]. It is of great importance that some deaf people process vibrations sensed via touch in the part of the brain used by other people for hearing. The emotional responses are stronger when different parts of the musical signals are delivered through different vibration elements to different locations on the subjects' back. Sign language and subtitles displays are very useful for our purpose as well. Furthermore the use of Haptic Chair when training deaf people to speak has a significant effect in enhancing the musical experience for them. There has been significant progress of sophisticated devices which use vibrations to stimulate parts of the body so as to create the perception of music for the deaf [11].

Captioning is one of the accessibility tools for persons who are hard of hearing and captions are the visual (text) representation of the sound track of a video, film, television program, or commercial [12]. In addition to dialog, captions include sound effects, speaker identification information, music notations, lyrics, and other key aural information. Accessibility is not to be confused with usability that is used to describe the extent to which a product (e.g., device, service, and environment) can be used by specified users to achieve specified goals with effectiveness, efficiency, and satisfaction in a specified context of use. The deaf persons are able to develop this innate 
visual literacy skill (sign language). In conclusion accessibility format in advertisement as a UD (Universal Design) is possible only in digital television [12].

The Kinect-Sign was tested in a Portuguese Sign-Language school and resulted in a joyful acceptance of the approach [13]. A serious game aims to teach sign-language and stands in a multi-dimensional area. The related work has to do with Natural User Interface, Serious Games - Sign Language Games (reproduction of sounds and music is involved) and Sign Language Recognition Algorithms. The latter is extensively studied and with the development of the Kinect sensor, it regained importance. Although most algorithms are not validated for a lexicon, there are already interesting solutions. Above all this paper proposes a system of a serious game in two modes: school and competition mode, not only for the deaf people but also for their friends, family members and colleagues [13].

The results indicated that the system called CAMLS could enhance hearing- impaired students' learning performance in a music course [14]. The aims are to implement it to support hearing-impaired students' music learning and to evaluate the usability of the system. The functions of CAMLS include: (i) the student tone differentiation measurement module, (ii) the tone tuning module and (iii) the song-learning module. The main purpose of musical tone-tuning module is to help hearing impaired students understand if a single played tone is correct or not. The students were trained with the main goal of being able to play a song with a flute in their music classes. Finally CAMLS improved students' interest, willingness to devote more time to music and improve one's music self-cognition [14].

These constraints of effective music perception are further compounded by the complexity of the acoustical features of music itself that require the perceptual integration of varying rhythmic, melodic, harmonic and timbral elements of sound [15]. It should be emphasized that music is approached here as arguably the most complex auditory stimulus in existence. The limitations of CI devices become evident when applied to music, particularly to inadequate spectral, fine-temporal, and dynamic range representation. Concluding we may say that it emphasizes to the deficits and inadequacies of CIs, as music is a very demanding challenge compared to speech. Further improvements and technological innovations of CI would offer a new dimension in auditory perception [15].

To our knowledge, there are no published data on rhythm perception in children with CI (Cochlear Implants) [16]. In the present study, a statistical difference was observed in rhythm discrimination between the $\mathrm{CI}$ and $\mathrm{NH}$ (normal hearing) groups of children. Basically the MuSIC (Musical Sounds in Cochlear Implants) test was developed to assess the music listening abilities of CI users. We should bear in mind that congenital deafness is different than acquired one. The CI users succeeded in identifying the original and instrumental versions of familiar recorded songs, but they could not identify the melody versions that were available in the original recordings [16].

An intelligent web based system overviews illustrates how learners and instructors interact with the system through online interactive environment [17]. Users can communicate synchronously through chat-rooms or asynchronously through e-mail and forums. Learners can access content areas which provide links for educational materi- 
als and can access utility areas that provide sets of services. All in all it presents the architecture of an intelligent web based learning system for digital circuits [17].

The learning game for the Deaf children was guided by the Multi-Tracks, subject of this investigation, which is a game to help the acquisition of a second language by deaf children [18]. The multimedia object is a game that consists of an outing in the city of Rio de Janeiro, initially starting at the three sets of three scenarios, each based on the actual objects and even musical sounds. The design related issues about Teaching-Learning include Selection, Comprehension, Transformation, Representation, Selection, Adaptation, Adjustment of Students' Characteristics, Teaching, Evaluation, Reflection and new ways to comprehend. Concluding, this project has elaborately investigated design activity and participation in teaching-learning processes [18].

\subsection{Learners with Motor Impairments or Physical disabilities}

Although the integration of students with and without disabilities in band would provide benefits for all, students who had experienced physical disabilities (eg. one hand player) could adapt themselves with little modification to play a different instrument that they used to [19]. Many musical instruments have been physically altered to serve players with disabilities. On the contrary a few adapted saxophones for amputees used technology and relied on electronic solenoids to depress the keys of the missing hand. Data from this study support and supplement the scant research previously reviewed regarding the obstacles band directors face when attempting to integrate students with physical disabilities into mainstream instrumental music study [19].

Concerning the presentation of PLAIME, there are three aims: 1. to explore the capability of the disabled students to acquire musical knowledge using PLAIME, 2. to measure the improvement in their ICT skills and 3. to observe their behavior along the study [20]. A wide variety of software tools for music learning is offered classified into five categories (Pellone 1992) like tutorials, drill and practice, game, simulation and problem solving. There is a detailed description of PLAIME referring to the goals of the platform, its structure, and the design of lectures, activities, and games. At the end of the program there was a great improvement in behavioral development and significant advance of music knowledge among the students [20].

There is an examination of gaze-based interaction methods, in which the novel 'Snap Clutch' software activates gaze control [21]. There was a test of the tool indicating that effective gaze control is possible. The basic method of interaction in (Massively Multiplayer Online Social Game) MMOSG of Second Life, MMOG environments is via a combination of mouse and keyboard with avatar movement normally being performed using cursor keys. Concluding it can be said that this work illustrates that gaze driven movement can potentially achieve parity with mouse and keyboard driven locomotion and it addresses as a viable modality to able-bodied and disabled users [21].

The movement-to-music (MTM) system is a radical computer system ideal for children with limited movements to play and even create music [22]. Having the MTM system at home is a beneficial experience for both parents and children. This 
system is quite affordable and effective because of its qualitative methodology. As a result, the MTM gives children the opportunity to expand their horizons. Nevertheless a much larger sample of children is required for better assumptions and conclusions on the issue [22].

Few of the adapted interfaces allow the music practice on acoustic instruments [23]. Playing acoustic music is a wonderful action but the disabled people with a lack of normal motor skills must adapt to with existing systems, using technical aids. Therefore a project called AE2M aims to solve this problem providing access to instrumental music for disabled children with the same autonomy level as non-disabled. The specific study illustrates the construction of a device which transforms any user environment object to a personalized interface, to play musical instruments [23].

Neuro-rehabilitation is not an exception to scientific revolution [24]. Rehabilitation specialists are working on designing and testing a great variety of rehabilitation systems and devices. Since traditional rehabilitation techniques are not enough for complete restore of patients with neurological disorders, virtual reality environments (VR) associated with mechatronic devices (robotics) offer a radical solution. The outcome will be an increased functional ability and increased participation in the community. In conclusion, the combination of VR and robotics opens new dimensions in rehabilitation which also means that augmented feedback can now be provided on a multitude of kinematic and dynamic parameters [24].

There is a classification of the right hand techniques into three areas strumming, string picking/skipping and string muting [25]. This study deals with the first area of strumming. The Actuated Guitar that utilizes a normal electrical guitar sensor to capture the rhythmic motion of alternative fully functioning limbs also becomes an effective device. Drawing on a range of inspiration it is obvious that disabilities do not obstruct people to experience usual instruments designed for non disabled people [25].

\section{Conclusions}

Realizing the enormous development of technological devices, it is quite natural to be used to deal with people with sensory and motor disabilities overcome their problems and communicate or interact with the surrounding world. Nevertheless there is extensive ongoing research about the great effects of technology in music therapy.

\section{$4 \quad$ References}

[1] Ranasinghe, C., Kavindu, Shyama C. Kumari, Dawpadee, B. Kiriella, and Lakshman, Jayaratne. "Adaptive Music Score Trainer for Visually Impaired in Sri Lanka." 24th Australasian Conference on Information Systems Adaptive Music Score Trainer for Visually Impaired, Melbourne (2013)

[2] Kiriella, Dawpadee B., Shyama C. Kumari, Kavindu C. Ranasinghe, and Lakshman Jayaratne. "Music Training Interface for Visually Impaired through a Novel Approach to Optical Music Recognition." GSTF J Comput 3.4 (2014) 
[3] Borges, José A., and Tomé D. "Teaching Music to Blind Children: New Strategies for Teaching through Interactive Use of Musibraille Software.", 5th International Conference on Software Development and Technologies for Enhancing Accessibility and Fighting Info-exclusion, DSAI, Procedia Computer Science, pp. 19-27 (2014)

[4] Lin, Chern S., Chi C. Lin, Shih-Wei Yang, Shuo-Rong Liou, and Yun-Long Lay. "A Musical Composition Assistant for the People with Severe Physical and Visual Disabilities." Assistive Technology 24.2 pp. 123-131 (2012)

[5] Sodnik, Jaka, Grega Jaku s, and Sašo Tomažič. "Multiple spatial sounds in hierarchical menu navigation for visually impaired computer users." International Journal of HumanComputer Studies 69.Vol. 1-2, pp. 100-112 (2011)

[6] Sánchez, Jaime. "A model to design interactive learning environments for children with visual disabilities." Educ Inf Technol 12.3, pp.149-163 (2007) https://doi.org/10.1007/ s10639-007-9039-3

[7] Drigas A.S., Koukianakis L.G., Papagerasimou Y. V. "A system for e-inclusion for individuals with sight disabilities." WSEAS TRANSACTIONS on CIRCUITS and SYSTEMS, Issue 11, Vol. 4, pp. 1776-1780 (2005)

[8] McGookin, D., Brewster St., and Jiang W. "Investigating touchscreen accessibility for people with visual impairments." Proceedings of the 5th Nordic conference on Humancomputer interaction building bridges - NordiCHI '08 (2008) https://doi.org/10.1145/ 1463160.1463193

[9] Evreinova, T. G., Evreinov, G. \& Raisamo, R. " A Case Study on Non-Visual Pen-Based Interaction with the Numerical Data." International Journal of Human Computer Interaction (IJHCI), Issue 2, Vol. 4 (2013)

[10] Bartlett, B., Estivill-Castro, V., Seymon, S., Tourky, A. "Robots for Pre-orientation and Interaction of Toddlers and Preschoolers who are Blind".

[11] Nanayakkara, S., Taylor E., Wyse L., Ong S. H. "An Enhanced Musical Experience for the Deaf: Design and Evaluation of a Music Display and a Haptic Chair." Proceedings of the 27th international conference on Human factors in computing systems - CHI 09 (2009) https://doi.org/10.1145/1518701.1518756

[12] Poothullil, J. M., Sahasrabudhe, S., Chavan, P. D. and Toppo D. "Captioning and Indian Sign Language as Accessibility Tools in Universal Design." SAGE Open 3.2 (2013)

[13] João, G., Cardoso T., and Rybarczyk Y. "Kinect-Sign, Teaching Sign Language to "Listeners" through a Game." Procedia Technology 17, pp. 384-391, (2014) https://doi.org/10.1016/j.protcy.2014.10.199

[14] Yang, H.-J., Lay, Y.-L., Liou, Y.-C., Tsao, W.-Y., and Lin, C.-K. "Development and evaluation of computer-aided music- learning system for the hearing impaired." Journal of Computer Assisted Learning 23.6, pp 466-476 (2007) https://doi.org/10.1111/j.13652729.2007.00229.x

[15] Limb, Charles J., and Alexis, T. Roy. "Technological, biological, and acoustical constraints to music perception in cochlear implant users". Hearing Research 308, pp 13-26 (2014) https://doi.org/10.1016/j.heares.2013.04.009

[16] Stabej, Katja K., Smid, L., Gros, A., Zargi, M., Kosir, A., and Vatovec, J. "The music perception abilities of prelingually deaf children with cochlear implants." International Journal of Pediatric Otorhinolaryngology 76.10, pp. 1392-1400 (2012) https://doi.org/10.1016/ j.ijporl.2012.07.004

[17] Elgaml, A. F., Baladoh, S. M. "An Intelligent Web-based System to Enhance Digital Circuits Concepts and Skills for Deaf Students." International Journal of Engineering and Innovative Technology (IJEIT) Vol. 4, Issue 2 (2014)

[18] Portugal, C., de Souza Couto, R. M. "Designing a Learning Game for the Deaf Children as an Educational Technology." Contemporary Educational Technology, 3(1), pp. 60-75 (2012) 
[19] Nabb, D. and Balcetis, E. "Access to Music Education: Nebraska Band Directors' Experiences and Attitudes Regarding Students With Physical Disabilities." Journal of Research in Music Education 57.4, pp. 308-319 (2009) https://doi.org/10.1177/0022429409353142

[20] Cano, M., \& Sanchez-Iborra, R. "On the use of a multimedia platform for music education with handicapped children: A case study." Computers \& Education, 87, pp. 254-276 (2015) https://doi.org/10.1016/j.compedu.2015.07.010

[21] Vickers, S., Istance, H. O., Hyrskykari A., Ali, N. and Bates, R. "Keeping an eye on the game: eye gaze interaction with Massively Multiplayer Online Games and virtual communities for motor impaired users." Proceedings of the 7th ICDVRAT with ArtAbilitation, Maia, Portugal, (2008)

[22] Tam, C., Schwellnus, H., Eaton, C., Hamdani, Y., Lamont, A., \& Chau, T. "Movement-tomusic computer technology: a developmental play experience for children with severe physical disabilities. Occupation Therapy International, 14(2), pp. 99-112 (2007) https://doi.org/10.1002/oti.227

[23] Veytizou, J., Xuereb, H., \& Thomann, G. "Design of a Clip Product Based on Customer Needs for Playing Acoustic Music. " Lecture Notes in Production Engineering, pp. 367376 (2013) https://doi.org/10.1007/978-3-642-30817-8 36

[24] Robertson, J. V., \& Roby-Brami, A. "Augmented feedback, virtual reality and robotics for designing new rehabilitation methods." Collection de L'Académie Européenne de Médecine de Réadaptation, pp. 223-245 (2010)

[25] Larsen, J. V., Overholt, D., \& Moeslund, T. B. "The Actuated Guitar: A platform enabling alternative interaction methods." In Proceedings of the Sound and Music Computing Conference 2013, Logos Verlag Berlin, pp 235-238 (2013)

[26] B. Aylett. "ROBOTS | Bringing intelligent machines to life?" ABC Books, Sydney NSW, Australia (2002)

[27] Pellone, G. "Developing instructional software". Australian Journal of Educational Technology, Vol. 8(1), pp. 65-68 (1992) https://doi.org/10.14742/ajet.2245

\section{Authors}

Theodorou Paraskevi has a MA in Special Education and a Msc in Digital Systems and Telecommunications. She is a Teaching Professional in Information and Communication Technologies (ICTs). She is also a Teacher of Music playing and the Theory of music. She has participated in various research projects regarding the use of ICTs in Special Education. (vantheodorou@sch.gr, van4.theodorou@gmail.com

Athanasios Drigas is a Research Director at IIT-N.C.S.R. Demokritos. He is the Coordinator of Telecoms Lab and founder of Net Media Lab since 1996. From 1990 to 1999 he was the Operational manager of the Greek Academic network. He has been the Coordinator of Several International Projects, in the fields of ICTs, and eservices (e-learning, e-psychology, e-government, e-inclusion, e-culture etc). He has published more than 270 articles, 7 books, 25 educational CD-ROMs and several patents. He has been a member of several International committees for the design and coordination of Network and ICT activities and of international conferences and journals. Also he has accepted several distinctions for his work (articles, projects, patents) (dr@iit.demokritos.gr).

Article submitted 01 November 2016. Published as resubmitted by the authors 23 January 2017. 